\title{
Analyse physique élémentaire de l'équation simplifiée du capteur plan à air en régime stationnaire
}

\author{
P. Rivet, Groupe Ramses \\ C.N.R.S. PIRDES, Laboratoire de Physique Corpusculaire, Collège de France, 75231 Paris Cedex 05, France
}

(Reçu le 28 septembre 1979, révisé le 17 janvier 1980, accepté le 23 janvier 1980)

\begin{abstract}
Résumé. - Un calcul relativement simple, effectué dans le cadre des approximations usuelles des capteurs solaires plans et appliqué au capteur à air, ou plus généralement à un capteur dont le fluide caloporteur s'écoule d'un mouvement uniforme au contact d'un absorbeur, permet de retrouver la formule classique donnant le rendement du capteur en explicitant la signification physique des paramètres qui interviennent dans cette formule. La conductivité de l'absorbeur est prise en compte dans le calcul et l'on s'aperçoit que son rôle est presque toujours négligeable. On donne enfin l'évolution des températures du fluide et de l'absorbeur le long du parcours du fluide.

Abstract. - A simple calculation made in the frame of approximations commonly used in the study of flat plate solar collectors and applied to the air collectors, allows to recover the usual formula giving the efficiency of the collector. The physical meaning of the parameters is explicitly exhibited. The conductivity of the absorbing surface is taken in account in the calculation. It has almost no part in the result. The temperature distribution of the fluid and of the absorbing surface in the flow direction are given.
\end{abstract}

L'analyse traditionnelle du capteur plan à eau (cf. par ex. réf. [3]) s'appuie sur un modèle comportant une série de tubes parallèles, entre lesquels le flux liquide, circulant dans un seul sens, se répartit également, solidaires physiquement et thermiquement d'une plaque absorbante rectangulaire.

Elle ne tient compte de la conductibilité de l'absorbeur que dans le sens transversal, lorsqu'il s'agit de conduire la chaleur de l'absorbeur aux tubes et au fluide. Elle néglige cette conductibilité lorsqu'elle entreprend le bilan énergétique d'une tranche de capteurs pour établir l'équation donnant la température du liquide en ce point de son parcours (réf. [3], 7.6.3) et en déduire le rendement du capteur (réf. [3] 7.7.5).

L'étude qui suit est fondée sur un modèle à une seule dimension $x$, constitué par un absorbeur de longueur $L$, de largeur $l$, en contact avec un fluide s'écoulant à vitesse uniforme dans la direction $x$, surmonté d'une vitre, modèle adapté aux capteurs à air ou à ruissellement.

Elle fait intervenir explicitement la conductibilité de l'absorbeur et permet d'en apprécier le rôle. Une bonne part des résultats doit pouvoir être étendue aux capteurs à eau de structure plus complexe.

Cette étude a aussi pour objet, dans une démarche analogue à celle d'autres auteurs (par ex. réf. [6]), de proposer pour les applications pratiques élémentaires, des expressions du rendement où la signification physique des paramètres est plus claire que dans la formule souvent employée (cf. réf. [1, 2, 4]) :

$$
\eta=\frac{\bar{\varphi}_{\mathrm{u}}}{\varphi_{0}}=A-B\left(\frac{\theta_{0}+\theta_{\mathrm{L}}}{2}-T_{\mathrm{ex}}\right)
$$

$\varphi_{0}$ flux solaire incident

$\bar{\varphi}_{\mathrm{u}}$ puissance moyenne extraite par unité de surface du capteur

$\theta_{0} \quad$ température d'entrée du fluide

$\theta_{\mathbf{L}}$ température de sortie du fluide

$T_{\text {ex }}$ température extérieure.

Notamment la température d'entrée du fluide y figure plutôt que la demi-somme des températures d'entrée et de sortie et l'influence du débit se lit facilement.

Cette étude reste élémentaire et ne prétend pas se substituer aux analyses plus complètes ou plus fines qui visent l'analyse de phénomènes complexes comme la sélectivité, la convection, les régimes transitoires, etc., indispensables pour faire progresser la technique des capteurs plans (par ex. réf. $[3,4,5]$ ).

Les approximations admises sont les suivantes :

- Approximation linéaire des termes de rayonne- 
ment en $\sigma T^{4}$ autour d'une valeur moyenne de référence $\hat{T}$ de la température.

- Effets de bord négligés.

- Caractéristiques optiques des milieux absorbeur et verre résumées dans des coefficients d'absorption $\alpha$, d'émission $\varepsilon$ pour l'absorbeur; de transmission $\tau$ pour le verre.

- Toutes les pertes thermiques vers l'extérieur affectant un élément de surface $\delta S$ prises en charge dans un seul terme $a\left(T-T_{\mathrm{ex}}\right) \delta S\left({ }^{1}\right)$ indépendant de la position de l'élément.

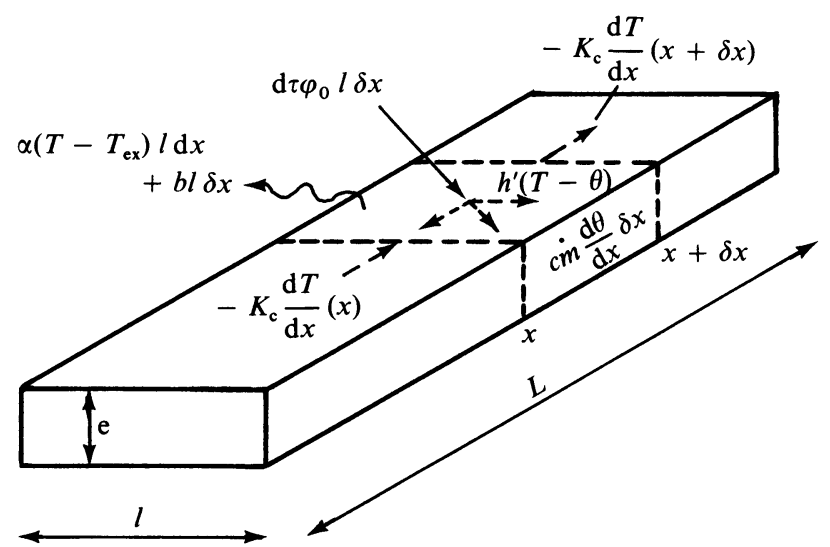

Fig. 1. - Bilan d'énergie d'un élément d'absorbeur et de fluide.

[Energy balance on collector and fluid element.]

L'équation d'équilibre thermique d'une tranche $\delta x$ d'absorbeur au voisinage du point $x$ s'écrit alors (Fig. 1) :

$$
\begin{aligned}
& \left(\alpha \tau \varphi_{0}-\varphi_{\mathrm{u}}\right) l \delta x= \\
& \quad=\left[a\left(T(x)-T_{\mathrm{ex}}\right)+b\right] l \delta x-K_{\mathrm{c}} \frac{\mathrm{d}^{2} T}{\mathrm{~d} x^{2}} l \delta x
\end{aligned}
$$

à laquelle il faut adjoindre les équations de transfert de la chaleur au fluide et d'extraction de la chaleur par le mouvement du fluide :

$$
\begin{gathered}
\varphi_{\mathrm{u}}=h^{\prime}(T(x)-\theta(x)) \\
\delta P_{\mathrm{u}}=\varphi_{\mathrm{u}} l \delta x=c \dot{m} \frac{\mathrm{d} \theta}{\mathrm{d} x} \delta x .
\end{gathered}
$$

( $\left.{ }^{1}\right) \mathrm{Si}$ on représente les pertes par conduction et convection du verre en direction de l'atmosphère par un coefficient de transfert $h$ les pertes par convection entre absorbeur et verre par $h_{1}$, alors

où

$$
a \simeq \frac{\left(\varepsilon \Sigma+h_{1}\right)(\Sigma+h)}{\varepsilon \Sigma+h_{1}+\Sigma+h}
$$

$$
\Sigma=4 \sigma \hat{T}^{3}
$$

Notations :

$\varphi_{\mathrm{u}}(x)$ énergie transmise de l'absorbeur au fluide par unité de surface au voisinage de $x$

$h^{\prime} \quad$ coefficient de transfert apparent correspondant

$T(x)$ température de l'absorbeur en $x$

$\theta(x)$ température du fluide en $x$

$K_{\mathrm{c}} \quad$ conductivité de l'absorbeur

$e \quad$ épaisseur de l'absorbeur

$\delta P_{\mathrm{u}} \quad$ puissance captée par le fluide entre $x$ et $x+\mathrm{d} x$

$\dot{m} \quad$ débit massique

$c \quad$ chaleur massique du fluide

$b$ terme correctif, en général négligeable au degré d'approximation où l'on opère, intervenant dans l'équation régissant les pertes thermiques vers l'extérieur.

1. Solution mathématique. - On emploiera cidessous les notations ', " etc... pour les dérivées successives par rapport à $x$ en estimant qu'il ne peut $\mathrm{y}$ avoir de confusion avec les paramètres affectés d'un indice prime.

En dérivant (I) par rapport à $x$ et en remplaçant $\varphi_{\mathrm{u}}^{\prime}$ par sa valeur tirée de (2)

$$
-h^{\prime}\left(T^{\prime}-\theta^{\prime}\right)=a T^{\prime}-e K_{\mathrm{c}} T^{\prime \prime \prime}
$$

puis

$\theta^{\prime}=\frac{l \varphi_{\mathrm{u}}}{c \dot{m}}=\frac{l}{c \dot{m}}\left(\alpha \tau \varphi_{\mathrm{o}}-a\left(T-T_{\mathrm{ex}}\right)-b+e K_{\mathrm{c}} T^{\prime \prime}\right)$.

On pose :

$$
T_{\mathrm{K}}=\frac{\alpha \tau \varphi_{0}}{a}+T_{\mathrm{ex}}-\frac{b}{a}
$$

donc :

$$
\begin{aligned}
-a u & =-a\left(T-T_{\mathrm{K}}\right)=-a\left(T-T_{\mathrm{ex}}\right)-b+\alpha \tau \varphi_{0} \\
T & =u+T_{\mathrm{K}}
\end{aligned}
$$

et

$$
\theta=T-\frac{\varphi_{\mathrm{u}}}{h^{\prime}}=T_{K}+\left(1+\frac{a}{h^{\prime}}\right) u-\frac{e K_{\mathrm{c}}}{h^{\prime}} u^{\prime \prime} .
$$

Les conditions aux limites

$$
T^{\prime}(0)=T^{\prime}(L)=u^{\prime}(0)=u^{\prime}(L)=0
$$

expriment l'isolement de l'absorbeur aux deux extrémités.

Revenant à l'éq. (4), on aboutit à une équation différentielle linéaire du $3^{\mathrm{e}}$ ordre sans second membre :

$$
-\frac{a l h^{\prime}}{c \dot{m}} u-\left(a+h^{\prime}\right) u^{\prime}+\frac{e K_{\mathrm{c}} l h^{\prime}}{c \dot{m}} u^{\prime \prime}+e K_{\mathrm{c}} u^{\prime \prime \prime}=0 .
$$


On introduit alors 3 paramètres dont le sens physique est assez clair :

$r=\frac{a}{h^{\prime}}$

rapport du coefficient global de pertes thermiques vers l'atmosphère au coefficient de transfert de l'absorbeur au fluide.

$X=\frac{\delta h^{\prime}}{c \dot{m}}=\frac{L l h^{\prime}}{c \dot{m}}$ rapport de la capacité de transfert de l'absorbeur au fluide à la capacité d'évacuation calorique du fluide; seul paramètre lié au débit.

$Y=\frac{h^{\prime} L^{2}}{e K_{\mathrm{c}}}$

qui compare le même coefficient de transfert à la capacité de transfert interne de l'absorbeur due à la conduction.

En écrivant la fonction résolvante sous la forme

$$
u=\exp \left(\lambda \frac{x}{L}\right)
$$

on parvient à l'équation du $3^{\mathrm{e}}$ degré :

$$
\lambda^{3}+X \lambda^{2}-(r+1) Y \lambda-r X Y=0 .
$$

La technique de résolution adoptée est celle de JahnkeEmde (par ex. réf. [7]). Elle fait appel à 3 paramètres intermédiaires

$$
\begin{aligned}
n & =\frac{1}{3}+(r+1) \frac{Y}{X^{2}} \\
m^{3} & =\frac{1}{27}+\frac{(1-2 r)}{6} \frac{Y}{X^{2}}
\end{aligned}
$$

La condition $k>1$ entraîne l'existence de 3 racines réelles; elle est toujours vérifiée dans notre cas. En outre il y a toujours une racine positive et deux négatives (somme et produit des racines).

Les solutions sont alors du type

$$
\lambda_{i}=\left(-0,33+m \lambda_{i}^{\prime}\right) X
$$

où les $\lambda_{i}^{\prime}$ sont tabulés en fonction de $k$. En fait il existe des expressions asymptotiques utilisables dès que $k \gtrsim 3$.

Connaissant $\lambda_{1}, \lambda_{2}, \lambda_{3}$ la démárche est la suivante : il faut déterminer les 3 coefficients $T_{1}, T_{2}, T_{3}$ de la solution

$u=T_{1} \exp \left(\lambda_{1} \frac{x}{L}\right)+T_{2} \exp \left(\lambda_{2} \frac{x}{L}\right)+T_{3} \exp \left(\lambda_{3} \frac{x}{L}\right)$.

Des conditions aux limites,

$$
\begin{aligned}
& u^{\prime}(0)=\lambda_{1} T_{1}+\lambda_{2} T_{2}+\lambda_{3} T_{3}=0 \\
& u^{\prime}(L)=\lambda_{1} T_{1} \mathrm{e}^{\lambda_{1}}+\lambda_{2} T_{2} \mathrm{e}^{\lambda_{2}}+\lambda_{3} T_{3} \mathrm{e}^{\lambda_{3}}=0
\end{aligned}
$$

on tire :

$$
\frac{T_{1}}{t_{1}}=\frac{T_{2}}{t_{2}}=\frac{T_{3}}{t_{3}}=s
$$

avec

$$
t_{i}=\lambda_{j} \lambda_{K}\left(\mathrm{e}^{\lambda_{j}}-\mathrm{e}^{\lambda_{K}}\right) \quad i, j, k=1,2,3 .
$$

Il se trouve que

$$
t_{2} \gg t_{1}, t_{3}
$$

On écrit alors :

$$
\begin{gathered}
u=s t_{2}\left[\tau_{1} \exp \left(\lambda_{1} \frac{x}{L}\right)+\exp \left(\lambda_{2} \frac{x}{L}\right)+\tau_{3} \exp \left(\lambda_{3} \frac{x}{L}\right)\right] \\
\tau_{1}=\frac{t_{1}}{t_{2}}, \quad \tau_{3}=\frac{t_{3}}{t_{2}} \cdots\left(\tau_{2}=1\right) .
\end{gathered}
$$

D'autre part, on se donne la température d'entrée du fluide $\theta(0)=\theta_{0}$ comme

$$
\begin{gathered}
\theta=T_{K}+(1+r) u-\frac{e K_{\mathrm{c}}}{h^{\prime}} u^{\prime \prime}=T_{\mathrm{K}}+s t_{2} \sum_{i}\left(1+r-\frac{\lambda_{i}^{2}}{Y}\right) \exp \left(\lambda_{i} \frac{x}{L}\right) \\
\theta_{0}-T_{K}=s t_{2} \sum_{i} \tau_{i}\left(1+r-\frac{\lambda_{i}^{2}}{Y}\right)=s t_{2} \sum \tau_{i}^{\prime}
\end{gathered}
$$

qu'on écrira sous la forme

$$
\theta_{0}-T_{K}=s t_{2}\left(1+r^{\prime}\right)
$$

alors

$$
\theta=T_{K}+\frac{\theta_{0}-T_{K}}{1+r^{\prime}}\left[\tau_{1}^{\prime} \exp \left(\lambda_{1} \frac{x}{L}\right)+\tau_{2}^{\prime} \exp \left(\lambda_{2} \frac{x}{L}\right)+\tau_{3}^{\prime} \exp \left(\lambda_{3} \frac{x}{L}\right)\right]
$$


L'expression qui se révèle la plus pratique est :

$$
\theta=\theta_{0}+\left(T_{K}-\theta_{0}\right)\left[1-\frac{1}{1+r^{\prime}}\left(\tau_{2}^{\prime} \exp \left(\lambda_{2} \frac{x}{L}\right)+\tau_{3}^{\prime} \exp \left(\lambda_{3} \frac{x}{L}\right)+\tau_{1}^{\prime} \exp \left(\lambda_{1} \frac{x}{L}\right)\right)\right] .
$$

On en déduit

$$
\Delta \theta=\theta_{\mathrm{L}}-\theta_{0}=\gamma\left(T_{\mathrm{K}}-\theta_{0}\right)
$$

avec

$$
\gamma=1-\frac{1}{1+r^{\prime}}\left(\tau_{2}^{\prime} \exp \left(\lambda_{2} \frac{x}{L}\right)+\tau_{3}^{\prime} \exp \left(\lambda_{3} \frac{x}{L}\right)+\tau_{1}^{\prime} \exp \left(\lambda_{1} \frac{x}{L}\right)\right)
$$

et donc

$$
\bar{\varphi}_{u}=\frac{P_{u}}{L l}=\frac{c \dot{m} \Delta \theta}{L l}=\frac{\gamma}{r X} a\left(T_{K}-\theta_{0}\right)=\frac{\gamma}{r X}\left(\alpha \tau \varphi_{0}-a\left(\theta_{0}-T_{\text {ex }}\right)-b\right)
$$

en négligeant le terme correctif $b$

$$
\eta=\frac{\gamma}{r X}\left(\alpha \tau-\frac{a\left(\theta_{0}-T_{\mathrm{ex}}\right)}{\varphi_{0}}\right)
$$

et enfin

$$
T=T_{K}+u=\theta_{0}+\left(T_{K}-\theta_{0}\right)\left[1-\frac{1}{1+r^{\prime}}\left(\exp \left(\lambda_{2} \frac{x}{L}\right)+\tau_{3} \exp \left(\lambda_{3} \frac{x}{L}\right)+\tau_{1} \exp \left(\lambda_{1} \frac{x}{L}\right)\right)\right] .
$$

Dans les applications pratiques $Y / X^{2}$ est assez grand.

Pour $Y / X^{2} \gtrsim 20$, on peut utiliser les expressions asymptotiques (car $k>3$ ) et on aboutit à des développements limités pour les $\lambda_{i}$

$$
\begin{gathered}
m \lambda_{1}^{\prime} \sim \sqrt{n}\left(1-\frac{1}{3 k \sqrt{3 k}}\right), \quad m \lambda_{2}^{\prime} \sim \frac{2 m^{3}}{n}, \quad m \lambda_{3}^{\prime} \sim \sqrt{n}\left(-1-\frac{1}{3 k \sqrt{3 k}}\right) \\
\lambda_{1}=\left(-0,33+m \lambda_{1}^{\prime}\right) X=\sqrt{(r+1) Y}-\frac{X}{2(r+1)}+\cdot \\
\lambda_{2}=\left(-0,33+m \lambda_{2}^{\prime}\right) X=X\left[-\frac{r}{r+1}+\frac{8 r-1}{27(r+1)^{2}}\left(\frac{Y}{X^{2}}\right)^{-1}+\cdot\right] \\
\lambda_{3}=\left(-0,33+m \lambda_{3}^{\prime}\right) X=-\sqrt{(r+1) Y}-\frac{X}{2(r+1)}+\cdot \\
t_{1} \sim \lambda_{2} \lambda_{3} \mathrm{e}^{\lambda_{2}}, \quad t_{2} \sim-\lambda_{3} \lambda_{1} \mathrm{e}^{\lambda_{1}}, \quad t_{3} \sim \lambda_{1} \lambda_{2} \mathrm{e}^{\lambda_{1}} \quad t_{2} \gg t_{3} \gg t_{1} .
\end{gathered}
$$

Dans la formule (7) donnant $u, \tau_{1}$ est négligeable

$$
\tau_{3}=\frac{t_{3}}{t_{2}} \sim-\frac{r}{(r+1)^{3 / 2}}\left(\frac{Y}{X^{2}}\right)^{-1 / 2}
$$

d'autre part

$$
\frac{\lambda_{1}^{2}}{Y} \sim r+1-\left((r+1) \frac{Y}{X^{2}}\right)^{-1 / 2}, \quad \frac{\lambda_{2}^{2}}{Y} \sim \frac{r^{2} X^{2}}{(r+1)^{2} Y}, \quad \frac{\lambda_{3}^{2}}{Y} \sim r+1+\left((r+1) \frac{Y}{X^{2}}\right)^{-1 / 2}
$$

$\tau_{1}^{\prime}$ est négligeable,

$$
\tau_{2}^{\prime} \sim 1+r-\frac{r^{2}}{(r+1)^{2}}\left(\frac{Y}{X^{2}}\right)^{-1}, \quad \tau_{3}^{\prime} \sim \frac{r}{(r+1)^{2}}\left(\frac{Y}{X^{2}}\right)^{-1}
$$

conduisant à

$$
\theta_{0}-T_{K}=s t_{2}\left(1+r^{\prime}\right) \text { avec } r^{\prime} \simeq r+\frac{r-r^{2}}{(r+1)^{2}}\left(\frac{Y}{X^{2}}\right)^{-1}
$$


Dans (9)

$$
\Delta \theta=\gamma\left(T_{K}-\theta_{0}\right) \quad \text { avec } \quad \gamma=1-\exp \left(-\frac{r X}{r+1}\right)+\frac{r}{(r+1)^{3}}\left(\frac{Y}{X^{2}}\right)^{-1} \exp \left(-\frac{r X}{r+1}\right)
$$

ce qui permet de calculer explicitement en se limitant au terme fondamental

$$
\bar{\varphi}_{u} \simeq \frac{1}{r X}\left(1-\exp \left[-\frac{r X}{r+1}\right]\right)\left(\alpha \tau \varphi_{0}-a\left(\theta_{0}-T_{\mathrm{ex}}\right)\right)
$$

comparable à (7.7.5 réf. [3]) et le rendement $\eta=\bar{\varphi}_{u} / \varphi_{0}$, formule où le débit figure à travers $X$.

La dépendance en $r$ n'est pas explicite à cause du terme en $a$ figurant dans $T_{K}$. On remarque en outre que le paramètre $Y$ n'intervient que dans un terme correctif dans l'expression de $\gamma$, c'est-à-dire que la conduction de l'absorbeur ne joue aucun rôle significatif tant que $Y / X^{2}$ est grand, cas usuel comme les exemples ci-dessous le prouvent.

Rappelons enfin les expressions (8) et (10) des températures de fluide et de l'absorbeur en fonction de $x$ qui deviennent ici, en allant jusqu'au $1^{\text {er }}$ ordre

$$
\begin{gathered}
\theta \simeq \theta_{0}+\left(T_{K}-\theta_{0}\right)\left[1-\exp \left(-\frac{r X}{r+1} \frac{x}{L}\right)-\frac{r}{(r+1)^{3}}\left(\frac{Y}{X^{2}}\right)^{-1} \exp \left(-\sqrt{(r+1) Y} \frac{x}{L}\right)\right] \\
T \simeq \theta_{0}+\left(T_{K}-\theta_{0}\right)\left[1-\frac{1}{r+1}\left(\exp \left[-\frac{r X}{r+1} \frac{x}{L}\right]-\frac{r}{(r+1)^{3 / 2}}\left(\frac{Y}{X^{2}}\right)^{-1 / 2} \exp \left[-\sqrt{(r+1) Y} \frac{x}{L}\right]\right)\right] .
\end{gathered}
$$

\section{Applications. -2.1 Domaine De Variation Des Paramètres.}

$r=\frac{a}{h^{\prime}} \quad$ Un capteur est d'autant meilleur que $r$ est petit $:$ il semble difficile actuellement de descendre audessous de 0,2 ou 0,3 . D'un autre côté, au niveau de $r=3$ ou 4 on atteint la limite tolérable de la médiocrité. La plage utile pourrait s'étendre de 0,2 à 3 . $X=\frac{L l h^{\prime}}{c \dot{m}} \quad \begin{aligned} & \text { Les débits très élevés sont sans intérêt et pour les débits très faibles, le gain en écart de température } \\ & \text { entre l'entrée et la sortie est payé par un rendement décroissant. La plage utile pourrait se situer }\end{aligned}$ entre 0,5 et 5 .

$Y=\frac{L^{2} h^{\prime}}{e K_{\mathrm{c}}}$ Pour le produit $e K_{\mathrm{c}}$, la limite supérieure est apportée par la conductivité des matériaux réels. Dans l'un des meilleurs cas, le cuivre, $K_{\mathrm{c}}=400 \mathrm{~W} / \mathrm{m}^{\circ} \mathrm{C}$, il faut $e=5 \mathrm{~mm}$ pour atteindre $e K_{\mathrm{c}}=2$. Une valeur de 0,1 peut constituer une moyenne.

$h^{\prime}$ doit être assez grand pour transmettre des flux de plusieurs centaines de $\mathrm{W} / \mathrm{m}^{2}$ sans écart de température tror grand entre absorbeur et fluide. On ne peut guère descendre au-dessous de 5 à $10 \mathrm{~W} / \mathrm{m}^{2}{ }^{\circ} \mathrm{C} ; \mathrm{L}$ est de l'ordre du mètre et ne peut dépasser quelques mètres. Ainsi les valeurs usuelles de $Y$ se situent autour de 2 à 400 . On se trouve très généralement dans des zones où $Y / X^{2}>20$ et où l'on peut donc appliquer les développements cidessus, en se limitant presque toujours aux premiers termes; les termes correctifs en $Y / X^{2}$ ne représentent en effet qu'au plus quelques $\%$ des termes principaux dans les différentes expressions. Ce sont alors les approximations faites dans le modèle qui limitent sa validité.

Exemple. - Capteur standard à air

$$
\begin{aligned}
\alpha \tau & =0,9 & & \varphi_{0}=800 \mathrm{~W} / \mathrm{m}^{2} \\
L & =2 \mathrm{~m} & & \theta_{0}=20^{\circ} \\
l & =1 \mathrm{~m} & & T_{\mathrm{ex}}=15^{\circ} \\
h^{\prime} & =20 \mathrm{~W} / \mathrm{m}^{2}{ }^{\circ} \mathrm{C} & & T_{K}=\frac{\alpha \tau \varphi_{0}}{a}+T_{\mathrm{ex}}=87^{\circ} \\
r & =\frac{a}{h^{\prime}}=0,5 & &
\end{aligned}
$$

I. - Absorbeur à faible conductivité

$$
e K_{\mathrm{c}}=0,1 \rightarrow Y=800 .
$$


a) Débit moyen $d=60 \mathrm{~m}^{3} / \mathrm{h} \rightarrow X=2, Y / X^{2}=200$

$$
\begin{aligned}
r X & =1 ; \quad \exp \left(-\frac{r X}{r+1}\right)=\mathrm{e}^{-0,66}=0,52 \\
\Delta \theta & =\left(1-\exp \left[-\frac{r X}{r+1}\right]\right)\left(T_{K}-\theta_{0}\right)=32^{\circ} \\
\eta & =\frac{1}{r X}\left(1-\exp \left[-\frac{r X}{r+1}\right]\right)\left(\alpha \tau-\frac{a\left(\theta_{0}-T_{\text {ex }}\right)}{\varphi_{0}}\right)=0,4 \\
\theta(x) & =\theta_{0}+\left(T_{K}-\theta_{0}\right)\left(1-\exp \left[-\frac{r X}{r+1} \frac{x}{L}\right]\right)=20^{\circ}+67^{\circ}\left(1-\exp \left[-0,66 \frac{x}{L}\right]\right) \\
T(x) & =20^{\circ}+67^{\circ}\left[1-0,66\left(\exp \left[-0,66 \frac{x}{L}\right]-0,02 \exp \left[-17 \frac{x}{L}\right]\right)\right] .
\end{aligned}
$$

b) Débit faible $d=20 \mathrm{~m}^{3} / \mathrm{h} \rightarrow X=6, Y / X^{2}=22,2$

$$
\begin{aligned}
r X & =3 ; \quad \exp \left(-\frac{r X}{r+1}\right)=\mathrm{e}^{-2}=0,135 \\
\Delta \theta & =0,86 \times 67^{\circ}=57^{\circ} \\
\eta & =0,29(0,9-0,06)=0,25 \\
\theta(x) & =20^{\circ}+67^{\circ}\left(1-\exp \left[-2 \frac{x}{L}\right]-0,007 \exp \left[-17 \frac{x}{L}\right]\right) \\
T(x) & =20^{\circ}+67^{\circ}\left[1-0,66\left(\exp \left[-2 \frac{x}{L}\right]-0,06 \exp \left[-17 \frac{x}{L}\right]\right)\right] .
\end{aligned}
$$

Les courbes $\theta(x), T(x)$ sont présentées sur la figure 2 .

II. - Absorbeur à forte conductivité

$$
e K_{\mathrm{c}}=2, \quad Y=40 .
$$

a) $d=60 \mathrm{~m}^{3} / \mathrm{h} \rightarrow X=2, Y / X^{2}=10$ (l'emploi des expressions asymptotiques est encore tolérable)

$$
\begin{aligned}
\Delta \theta & =32^{\circ} \\
\eta & =0,4 \\
\theta(x) & =20^{\circ}+67^{\circ}\left(1-\exp \left[-0,66 \frac{x}{L}\right]-0,015 \exp \left[-8,3 \frac{x}{L}\right]\right) \\
T(x) & =20^{\circ}+67^{\circ}\left[1-0,66\left(\exp \left[-0,66 \frac{x}{L}\right]-0,09 \exp \left[-8,3 \frac{x}{L}\right]\right)\right] .
\end{aligned}
$$

b) $d=20 \mathrm{~m}^{3} / \mathrm{h} \rightarrow X=6, Y / X^{2}=1,11$

$$
\begin{aligned}
& \left.\begin{array}{rl}
n & =0,33+1,5 \times 1,11=1,98 \\
m^{3} & =0,037, m=0,333, m^{2}=0,111
\end{array}\right\} \quad k=5,94 \\
& \sqrt{n}=1,4 \\
& m \lambda_{1}^{\prime}=1,38, \quad m \lambda_{2}^{\prime}=0,037, \quad m \lambda_{3}^{\prime}=-1,41 \\
& \lambda_{1}=6,3, \quad \lambda_{2}=-1,76, \quad \lambda_{3}=-10,4 \\
& \tau_{1} \mathrm{e}^{\lambda_{1}} \sim-\frac{\lambda_{2}}{\lambda_{1}} \mathrm{e}^{\lambda_{2}} \sim 0,047, \quad \tau_{2}=1, \quad \tau_{3}=-\frac{\lambda_{2}}{\lambda_{3}}=-0,17 \\
& \tau_{1}^{\prime} \mathrm{e}^{\lambda_{1}}=0,024, \quad \tau_{2}^{\prime}=1,42, \quad \tau_{3}^{\prime}=0,2 \\
& 1+r^{\prime}=\Sigma \tau^{\prime}=1,62, \quad \gamma=0,84 \rightarrow \Delta \theta=55^{\circ}, \quad \eta=0,24 \\
& \theta(x)=20^{\circ}+67^{\circ}\left[1-0,62\left(1,42 \exp \left[-1,77 \frac{x}{L}\right]+0,2 \exp \left[-10,4 \frac{x}{L}\right]\right)\right] \\
& T(x)=20^{\circ}+67^{\circ}\left[1-0,62\left(\exp \left[-1,77 \frac{x}{L}\right]-0,17 \exp \left[-10,4 \frac{x}{L}\right]\right)\right] \text {. }
\end{aligned}
$$

Les courbes correspondantes sont données sur la figure 3. 


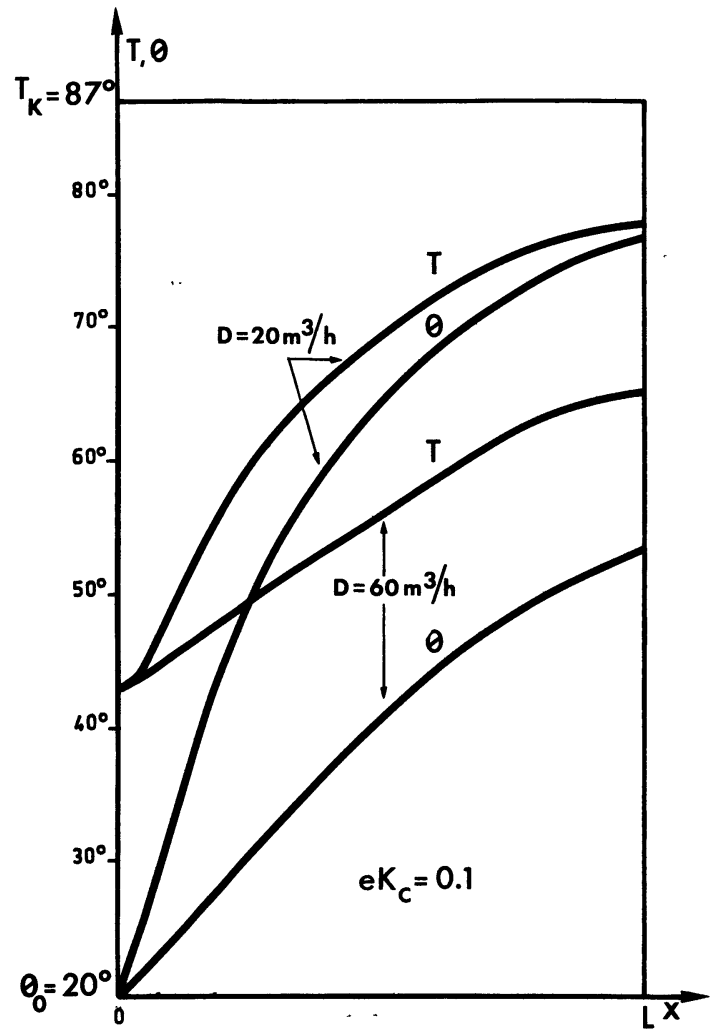

Fig. 2. - Evaluation des températures de l'absorbeur $(T)$ et de l'air $(\theta)$ le long du parcours de l'air pour un absorbeur de faible conductivité $e K_{\mathrm{c}}=0,1$. Deux débits sont envisagés $D=20 \mathrm{~m}^{3} / \mathrm{h}$ et $60 \mathrm{~m}^{3} / \mathrm{h}$.

[Distribution of the temperature of the absorbing surface $(T)$ and the air $(\theta)$ in flow direction. For a weakly conducting absorbing surface $e K_{\mathrm{c}}=0.1$. Two flow rate are considered $D=20 \mathrm{~m}^{3} / \mathrm{h}$ and $60 \mathrm{~m}^{3} / \mathrm{h}$.]

3. Conclusion. - L'étude précédente montre que dans la plupart des cas le transfert de chaleur longitudinal (parallèlement au parcours du fluide) par conduction dans l'absorbeur ne joue aucun rôle, sauf dans des cas marginaux combinant faible débit et conduction élevée où les courbes $T(x)$ et $\theta(x)$ sont un peu déformées au voisinage des deux extrémités $x=0$ et $x=L$.

Bien que le calcul ci-dessus soit relatif à un modèle conforme au capteur à air, les conclusions peuvent qualitativement s'étendre à un capteur à tubes d'eau

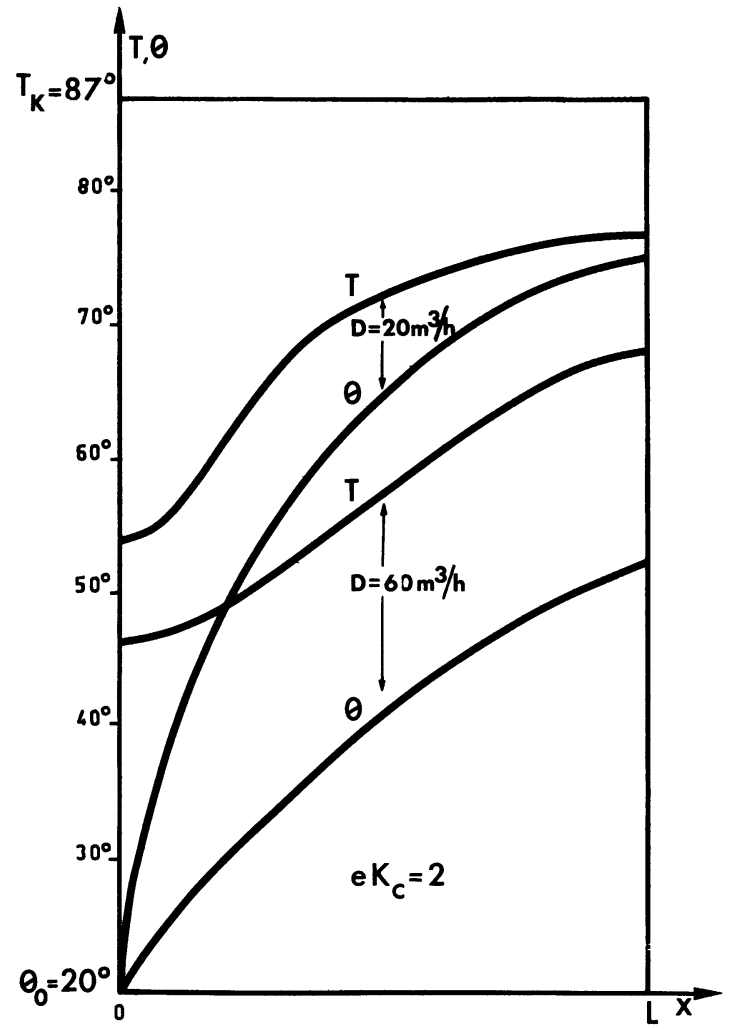

Fig. 3. - Evolution des températures de l'absorbeur $(T)$ et de l'air $(\theta)$ le long du parcours de l'air pour un absorbeur de conductivité élevée $e K_{\mathrm{c}}=2$. Deux débits sont envisagés $D=20 \mathrm{~m}^{3} / \mathrm{h}$ et $60 \mathrm{~m}^{3} / \mathrm{h}$.

[Distribution of the temperature of the absorbing surface $(T)$ and the air $(\theta)$ in flow direction for an highly conducting absorbing surface $e K_{\mathrm{c}}=2$. Two flow rate are considered $D=20 \mathrm{~m}^{3} / \mathrm{h}$ and $60 \mathrm{~m}^{3} / \mathrm{h}$.]

longitudinaux et légitimer l'hypothèse suivant laquelle le transfert de chaleur longitudinal est en général négligeable. En effet si l'on revient au bilan d'énergie de l'élément d'absorbeur $\delta x$ (éq. (1)), le résultat obtenu signifie que l'apport de chaleur par conduction est faible, en valeur absolue, devant les transferts représentés par l'apport solaire, et la chaleur transmise au fluide; il en est de même par principe pour un bon capteur à eau sauf dans le cas extrême où un très faible débit réduit le transfert au fluide et où simultanément une bonne conductivité permet à la chaleur de circuler dans le sens longitudinal.

\section{Bibliographie}

[1] Chouart, Ph., Berhondo, P., Rapport ESD/AEE, HE 112 T 261 (1976).

[2] Berhondo, P., Rapport EDF, HC 312 W8 33 (1975).

[3] DufFIE and BeCKmanN, Solar Energy thermal processes (John Wiley and sons) 1974.

[4] Devin, B., Analyse physique et expérimentale des capteurs plans en régime statique. Compte rendu Ecole d'été de Cargèse, 1977. Energie solaire. Conversion et applications (Editions du C.N.R.S.).
[5] PERI, G., Les capteurs convertisseurs héliothermiques sous concentration (Cahier AFEDES, $\mathrm{n}^{\circ}$ 4).

[6] Sacadura, J. F. O., Equation caractéristique des capteurs solaires plans sans concentration, Revue générale de thermique $\mathrm{n}^{\circ} 171$ (Mars 1976).

[7] BoLl, M., Tables numériques universelles (Dunod), p. 705-708. 